\title{
A Compliant Multi-finger Grasp Approach Control Strategy Based on the Virtual Spring Framework
}

\author{
Zhaopeng Chen ${ }^{(凶)}$, Christian Ott, and Neal Y. Lii \\ Institute of Robotics and Mechatronics, German Aerospace Center, DLR, \\ 82234 Wessling, Germany \\ zhaopeng. chen@dlr.de
}

\begin{abstract}
This paper presents an adaptive compliant multi-finger grasp approach control strategy based on based on a new interpretation of the virtual spatial spring framework, to improve the grasp performance for target objects with position errors. An n-finger virtual spatial spring frame is proposed to achieve the adaptive compliant grasp control. Twofinger grasp control based on a single virtual spring is tackled, and then extended to multi-finger grasp control. Virtual springs for self-collision avoidance among digits are constructed to form the complete adaptive compliant grasp control law. With the virtual-spring based adaptive compliant grasp approach control strategy, the first robot finger to experience unexpected impact remains in contact with the object, while the rest of the fingers are continuously, adaptively driven toward re-adjusted grasping positions by the virtual springs without the need for on-line replanning. Experimental results demonstrate effectiveness of the virtual-spring based grasp controller, and significantly larger position errors of the target object can be accommodated with the proposed adaptive compliant grasp control strategy.
\end{abstract}

\section{Introduction}

Multi-fingered dexterous robotic hands differ from two-jaw grippers and underactuated hands in the variety of grasp types it can achieve, and the unique capability of in-hand manipulation. Due to the multi-finger contact with the object, the object in-hand pose can also be more effectively estimated to help facilitate improved execution of the follow-up task such as object manipulation. However, the grasp quality and manipulation performance of multi-fingered robotic hands rely on grasp planning algorithms based on known object model or information [1], which are limited by modeling and control errors. In scenarios where objects are not in the expected location for the robot, or the end-effector of the robot is not in the expected configuration as the robot is commanded, unexpected contacts or collisions caused by the uncertainties during the grasping task execution can result in grasp failure or poor grasp quality. This in turn hinders the performance of executing object manipulation tasks further down the task chain. 
The reduced grasp and manipulation performance while encountering object location errors have been observed in our previous work [2].

In order to improve the performance during grasp execution, reactive grasp control strategies, and post-grasp object estimation have been investigated in the recent years. Takahashi et al. [3] proposed a robust parallel force/position control based on tactile feedback, in order to address object grasp tasks with unknown stiffness and shapes. Experimental results demonstrated the effectiveness of the control strategy. However, object location uncertainties were not addressed in this work. A contact-reactive grasping method was presented by Hsiao et al. [4], for partially modelled objects. Promising results were obtained with PR2 personal robot, for which a parallel two-jaw gripper was utilized in this work, rather than multi-fingered dexterous robotic hands. Chalon et al. [5] proposed an in-hand object location estimation algorithm using a Particle Filter. However, object pose in post-grasp phase was investigated, rather than grasp execution phase.

Hogan [6] introduced the impedance control framework, which is adopted in this paper to achieve a unified grasp approach strategy, including contact detection, reactive control during contact, and following adaptive grasp approach control. Khatib [7] analyzed the inertial properties of the object level manipulation, and Fasse et al. [8] introduced a object level impedance controller based on the virtual spatial spring. The first attempt to apply the virtual spatial spring system into multi-finger manipulation was made by Wimböck et al. [9]. And experiments were conducted on the 4-fingered dexterous robotic hand DLR II. However, the above works present spatial virtual spring based impedance controllers are mainly designed for objects manipulation in post-grasp phase. The virtual spatial spring concept [10] is utilized in this paper, in order to obtain an adaptive compliant grasp control scheme. In this paper, the impedance control and spatial virtual spring concept will be utilized to achieve adaptive compliant control in grasp approach phase.

To adaptively grasp objects with un-known properties, passive compliance is widely investigated in underactuated robotic hands [11]. The passive adaptive behavior of the fingers allows them to wrap around the object without needing sensing or control, thus obtaining stable power grasps [12]. However, those approaches tend to have limited or no in-hand manipulation capabilities, as they are not able to control individual degrees of freedom in the fingers. And the unmodeled information, such as object location errors and un-expected contacts can not be detected, because of the lack of sensory feedback. Recently, the possibility of obtaining basic manipulation capabilities with underactuated hands has been explored [13]. In contrast to those works, the proposed framework endows a multi-fingered hand with the ability to adapt the grasp approach according to the actual environment, thus creating a system robust to pose uncertainties in the object location with respect to the hand, while still keeping the capability of in-hand manipulation that fully actuated multi-fingered hands provide.

This paper is organized as the following: Section 2 describes the adaptive compliant grasp approach strategy; Section 3 presents the adaptive multi-finger 
compliant grasp controller based on the virtual spatial spring framework, including two-finger case and multi-finger case; control and grasp experimental results are presented in Section 4. Finally, the conclusions of this work are drawn and presented in Section 5.

\section{Framework of the Adaptive Compliant Grasp Approach Strategy}

Location uncertainties of the target objects are one of the main obstacle for autonomous grasp task execution with multi-fingered dexterous robotic hands. In order to address this problem, an adaptive compliant grasp approach control strategy, which address the hand's approach toward the object, up until the state of a stable grasp, is proposed in this paper as shown in Fig. 1. The adaptive compliant grasp approach strategy is composed of the following sequential phases:

(a) Adaptive compliant grasp

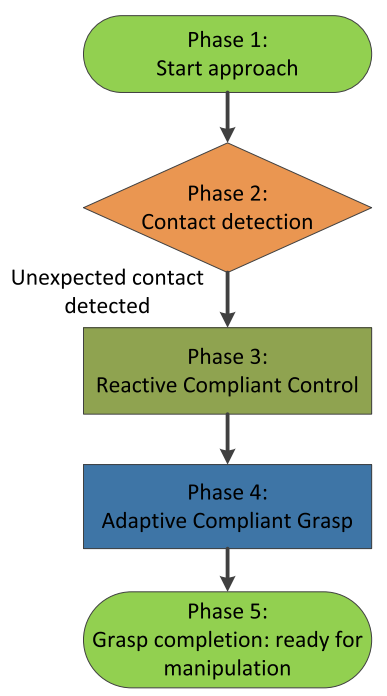

(b) Non-adaptive grasp

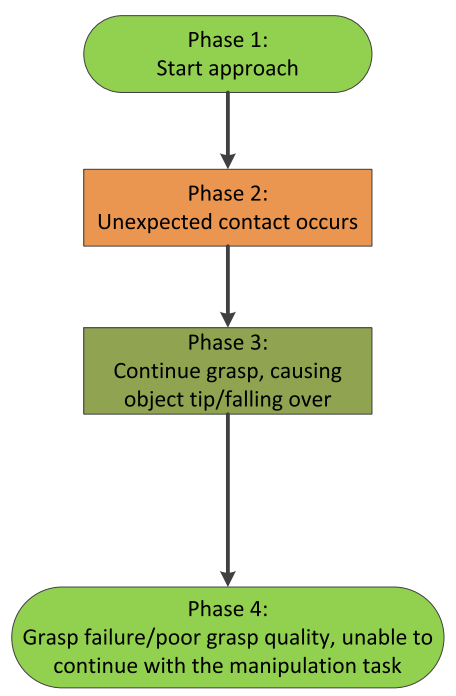

Fig. 1. Comparison of the adaptive and non-adaptive grasp approach concepts. As the hand starts its approach toward the target object (phase 1 in (a) and (b)), one finger would make a first contact unexpectedly due to the object position error (phase 2 in (a) and (b)). In the non-adaptive grasp approach, all fingers continue to move toward the expected object position((b) phase 3$)$. This can cause the object tipping, or falling over in some cases, which results a grasp failure ((b) phase 4). On the other hand, the proposed adaptive compliant grasp approach would keep the first-contact finger in compliant contact with the object ((a) phase 3), while using virtual springs to draw the other fingers to the adjusted desired position((a) phase 4$)$. The remaining fingers would then move in until every finger makes contact with the object to complete the grasp ((a) phase 5). 
Phase 1: Grasp approach starts. All the robotic fingers are controlled to approach the desired positions individually, with a Cartesian impedance controller. The desired positions and control parameters are obtained through an off-line grasp planner;

Phase 2: Contact detection. When unexpected contact occurs (the joint torque sensor reaches the specified threshold), displacement of the target object is detected by the joint torque sensor integrated in the robot finger;

Phase 3: Reactive compliant control. The reactive compliant control strategy is employed to stop the finger motion in a compliant way to reduce the impact force, so that the unplanned motion of the object due to the impact force is minimized;

Phase 4: Adaptive compliant grasp. Once the contact is detected, fingers without contact is controlled towards a locally adjusted grasp position, through an compliant grasp approach control strategy based on spatial virtual spring concept, until the target object is grasped.

Phase 5: Grasp complete. The target object is stably grasped, and the robotic hand/arm prepares for further manipulation tasks.

During the approach of Phase 1, a joint-torque based Cartesian impedance controller is utilized to drive the robot finger joints [14]. Once the first unexpected contact is detected by the joint torque sensor(when the joint torque reaches the threshold, Phase 2), the finger in contact with the object is required to remain with respect to the object in a compliant way (Phase 3) so that the unplanned motion of the object is minimized. The compliant behavior of the robot finger during contact is realized as:

- $q_{d}(t)=q(t)\left(t \geq t_{c}\right)$, where $t_{c}$ represents the time instant of the contact.

- $k_{p}=0\left(t \geq t_{c}\right)$, where $k_{p}$ indicates the stiffness of the joint impedance controller.

While the finger with the first contact remains in contact with the object upon contact detection with reactive compliant control, the adaptive compliant grasp control in Phase 4 is activated so that the rest fingers are driven towards the adjusted desired position by the spatial virtual springs. With respect to the first finger in contact, the non-contact fingers are automatic guided to the adjusted grasp position, even with the unplanned motion of the object caused by the contact forces. There are no vision involved in the grasp approach control strategy, and no on-line planner or re-grasp behaviors are required here to complete the grasp execution. The proposed adaptive compliant grasp approach control based on spatial virtual springs will be discussed in more detail in the following section.

Our experimental setup is based on the dexterous multisensory robotic hand DLR/HIT II [15][16]. The torque sensors integrated in joints of the hand are utilized to detect unexpected contact between the finger and the object in Phase 2. The available sensitivity of the contact detection is mainly determined by noise of the joint toque sensor, which is less than $0.01 \mathrm{~N} \cdot \mathrm{m}$. 


\section{The Multi-fingered Adaptive Compliant Grasp Approach Control}

\subsection{The n-Finger Virtual Spatial Spring Frame}

In order to achieve adaptive compliant grasp approach in Phase 4 as discussed in the last section, a n-finger $(n \geq 2)$ spatial virtual spring frame is proposed in this paper. As illustrated in Fig. 2, the virtual-spring frame is composed with $n-1$ spatial virtual springs attached between the thumb and each of the rest fingers on the robotic hand, and $n-2$ collision-avoidance virtual springs between adjacent fingers(except the thumb). The composition of n-finger virtual-spring frame in this paper rely on $n$ individual Cartesian positions of the robot fingers, rather than combined ones as previously proposed in [16]. This format of virtual-spring frame reduces couplings resulted from combinations of finger positions, and considers self-collision avoidance as an intrinsic feature of the framework, which improve the dynamic behavior and robustness of the virtual-spring frame based compliant control, and thus pave the way towards realizing adaptive compliant grasp approach based on the multi-finger virtual-spring frame.

When un-expected contact is detected by any of the fingers, the virtual springs drive the rest of the fingers to the adjusted desire positions. And proper distances between adjacent fingers can be obtained through repulsive forces from collision-avoidance springs during the grasp execution. The object is actually "caged" into the robotic fingers controlled by the closed form virtual-spring frame. The forming of the virtual-spring frame and grasping force can be defined through grasp preshape selection algorithms [1][17] based on the information of

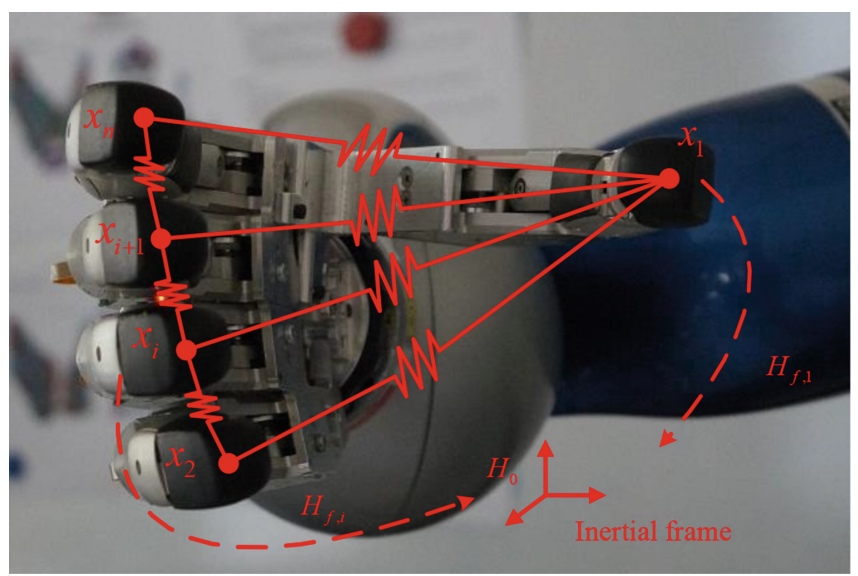

Fig. 2. The n-finger spatial virtual spring framework with self-collision avoidance springs between adjacent fingers on the DLR/HIT II dexterous robotic hand. $x_{1}$ and $x_{i}$ represent the Cartesian positions of the $1^{\text {st }}$ finger (the thumb) and the $i^{\text {th }}$ finger, respectively. $H_{1 i}$ and $H_{f i}$ denote the body coordinates of the thumb and the $i^{\text {th }}$ finger, respectively. $H_{0}$ indicates the inertial frame. 
the target objects. In this way, the rest length of the virtual springs and stiffness are chosen in grasp selection phase before approaching (Phase 1). It should be noted that this paper considers only precision grasps and the point contact with friction (PCWF) model.

\subsection{The Simple Case: Adaptive Compliant Grasp Approach Control with a Two-Finger Virtual Spatial Spring}

Since the complete virtual-spring frame is composed of several virtual spatial springs between the thumb( $1^{\text {st }}$ finger $)$ and one of the rest fingers $\left(i^{t h}\right)$, a single arbitrary two-finger spatial virtual spring case is tackled in this section, not only for the demonstration of the fundamental concept of the adaptive grasp controller presented in this paper, but also for a large portion of actual autonomous grasp tasks. The adaptive grasp controller will be extended to multi-finger grasping scenario in the next section.

The two-finger adaptive compliant grasp controller is based on the Cartesian position of the two robot fingers, as shown in Fig. 2. The compliant grasp approach control law based on the virtual spatial spring can be written as:

$$
\boldsymbol{\tau}_{a d a}=-\frac{\partial V_{1 i}(\boldsymbol{\theta})}{\partial \boldsymbol{\theta}^{T}}-\boldsymbol{D}_{1 i}(\boldsymbol{\theta}) \dot{\boldsymbol{\theta}}+g(\boldsymbol{\theta})
$$

where the actuator torque vector, $\tau_{a d a}$, is considered as the control input. $V_{1 i}$ is the energy stored in the virtual spatial spring. $\boldsymbol{\theta}$ indicates the vector of the joint angle. $D_{1 i}(\boldsymbol{\theta})$ represents the damping term with respect to the joint space, which is mapped from the damping force along the virtual spring direction. $g(\boldsymbol{\theta})$ is the gravity term. Based on passivity control theory, the energy store function of the virtual spatial spring can be chosen as:

$$
V_{1 i}=\frac{1}{2} K_{1 i}\left(\left\|\boldsymbol{\Delta} \boldsymbol{x}_{1, i}\right\|-l_{o}\right)^{2}
$$

where $\boldsymbol{\Delta} \boldsymbol{x}_{1 i}=\left\|\boldsymbol{x}_{1}-\boldsymbol{x}_{i}\right\|$ represents the distance between the center points of the two fingertips, while $l_{o}$ indicates the rest length of the virtual spatial spring, which is defined depending on the size of the object. $K_{1 i}$ indicates the stiffness parameter of the virtual spring. $x_{1}$ and $x_{i}$ are the Cartesian position of fingertips. The control force generated by the virtual spatial spring, which is in the virtual spring direction, can be mapped into joint space with a general Jacobian matrix $J_{1 i}$ as:

$$
\begin{aligned}
\boldsymbol{\tau}_{1 i} & =-\boldsymbol{J}_{1 i}^{T} \underbrace{K_{1 i}\left(\boldsymbol{\Delta} \boldsymbol{x}_{1, i}-l_{o}\right)}_{\boldsymbol{F}_{1 i}} \\
\boldsymbol{J}_{1 i} & =\frac{\boldsymbol{\Delta} \boldsymbol{x}_{1, i}}{\left\|\boldsymbol{\Delta} \boldsymbol{x}_{1, i}\right\|} \frac{\partial \boldsymbol{\Delta} \boldsymbol{x}_{1, i}}{\partial \boldsymbol{x}_{1 i}^{T}}\left(\boldsymbol{x}_{1}, \boldsymbol{x}_{i}\right)\left[\begin{array}{cc}
\boldsymbol{J}_{1} & 0 \\
0 & \boldsymbol{J}_{i}
\end{array}\right]
\end{aligned}
$$

where $\boldsymbol{J}_{1}$ and $\boldsymbol{J}_{i}$ represent the Jacobian matrices mapping coordinates $\boldsymbol{H}_{1}$ and $\boldsymbol{H}_{i}$ into the joint space of the fingers, respectively. The adaptive grasp controller 
can be derived together with an appropriately designed damping term $D_{1 i}$ :

$$
\boldsymbol{\tau}_{a d a}=-\boldsymbol{\tau}_{1 i}-\underbrace{\boldsymbol{J}_{1 i}^{T} \boldsymbol{D}_{1 i} \boldsymbol{J}_{1 i}}_{\boldsymbol{D}_{1 i}(\boldsymbol{\theta})} \dot{\boldsymbol{\theta}}
$$

where the damping term $\boldsymbol{D}_{1 i}$ is achieved by the Double Diagonalization approach[18].

\subsection{The Multi-fingered Adaptive Compliant Grasp Approach Control Strategy}

The multi-finger adaptive compliant grasp approach controller can be deduced from the derivation of two-finger adaptive grasp approach control law based on a single spatial spring, which is presented in the last section. By summing all the energy storage functions of the virtual spring framework, the multi-finger adaptive compliant grasp approach control law can be written as:

$$
\boldsymbol{\tau}_{a d a m}=-\frac{\partial V(\boldsymbol{\theta})}{\partial \boldsymbol{\theta}}-\boldsymbol{D}(\boldsymbol{\theta}) \dot{\boldsymbol{\theta}}+g(\boldsymbol{\theta})
$$

where $\boldsymbol{\tau}_{\text {adam }}$ represents the control input vector. $\boldsymbol{D}(\boldsymbol{\theta})$ and $g(\boldsymbol{\theta})$ are the damping term and gravity term, respectively. The energy storage function of the complete virtual spatial spring system $V$ can be defined as:

$$
V=\sum_{i=2}^{n} V_{1 i}
$$

where the $V_{1 i}$ is the energy storage function of the spatial spring between the thumb $\left(1^{s t}\right)$ and one of the rest fingers $\left(i^{t h}\right)$. $n$ indicates the number of the fingers involved in the grasp task. Then the multi-finger adaptive grasp control law can be represented as:

$$
\boldsymbol{\tau}_{a d a m}=-\sum_{i=2}^{n} \boldsymbol{\tau}_{1 i}^{n}-\sum_{i=2}^{n}\left(\boldsymbol{D}_{1 i}^{n}(\boldsymbol{\theta}) \dot{\boldsymbol{\theta}}\right)
$$

where $\boldsymbol{\tau}_{\text {adam }}$ represents the actuator torque vector as control input of the multifinger adaptive compliant control law. $\tau_{1 i}$ is described as equation (3).

\subsection{Self-Collision Avoidance Between Adjacent Fingers}

In order to prevent self-collisions among the digits, virtual springs between adjacent fingers are constructed so that not only the forming of the virtual-spring based grasp is maintained as the selected pre-shape during the whole grasp approach phases, but also repulsive forces are generated if the fingers are within the range of minimum safe distance, as shown in Fig. 2. The energy storage function 
of the self-collision avoidance virtual springs between adjacent fingers can be written as:

$$
V_{i}^{c}= \begin{cases}\frac{1}{2} K_{i}^{c}\left(l_{i}-l_{\text {safe }}\right)^{2} & l_{i} \leq l_{\text {safe }} \\ 0 & l_{i}>l_{\text {safe }}\end{cases}
$$

where $l_{i}=\left\|x_{i}-x_{i+1}\right\|$ indicates the distance between the $i^{\text {th }}$ and $(i+1)^{t h}$ finger, and $l_{\text {safe }}$ represents the minimum safe distance. $K_{i}^{c}$ defines the stiffness of the self-collision avoidance virtual spring. The self-collision avoidance control law can be expressed as:

$$
\boldsymbol{\tau}_{c o l}=-\sum_{i=2}^{n-1} \boldsymbol{\tau}_{i}^{c}-\sum_{i=2}^{n-1} \underbrace{\boldsymbol{J}_{i}^{c T} \boldsymbol{D}_{i}^{c} \boldsymbol{J}_{i}^{c}}_{\boldsymbol{D}_{i}^{c}(\boldsymbol{\theta})} \dot{\boldsymbol{\theta}}
$$

where:

$$
\boldsymbol{\tau}_{i}^{c}= \begin{cases}-\boldsymbol{J}_{i}^{c T} K_{i}^{c}\left(l_{i}-l_{\text {safe }}\right) & l_{i} \leq l_{\text {safe }} \\ 0 & l_{i}>l_{\text {safe }}\end{cases}
$$

$\boldsymbol{J}_{i}^{c}$ represents the general Jacobian matrix, as described in equation (3). $\boldsymbol{\tau}_{\text {col }}$ represents the actuator torque vector as control input of the self-collision avoidance control law.

Together with the negative joint torque feedback and self-collision avoidance controller, the complete adaptive grasp control law can be expressed as:

$$
\boldsymbol{\tau}_{\text {complete }}=\boldsymbol{K}_{\tau}\left(\boldsymbol{\tau}_{\text {adam }}+\boldsymbol{\tau}_{\text {col }}\right)+\left(\boldsymbol{I}-\boldsymbol{K}_{\tau}\right) \boldsymbol{\tau}
$$

where $\boldsymbol{\tau}_{\text {adam }}$ and $\boldsymbol{\tau}_{c o l}$ are the command joint torque vectors of the adaptive compliant grasp approach controller, and the self-collision avoidance controller, respectively. $\boldsymbol{\tau}$ indicates the external torque on joints of the robot finger, and $\boldsymbol{K}_{\tau}$ is a diagonal matrix representing the torque feedback gain, which contains $k_{\tau i} \geq$ 1 and determined by the noise level of the torque sensor. $\boldsymbol{K}_{\tau}$ actually defines the reduction ratio of the robot finger inertia reacting to external forces/torques.

\section{Experimental Results and Analysis}

\subsection{Experimental Evaluation of the Adaptive Compliant Control Based on the Multi-finger form Virtual Spring Framework}

Experiments of 3-finger form adaptive compliant grasp control are conducted, in order to evaluate the control law proposed in Section 3. During the experiment, operator pushes the thumb to simulate an unexpected contact with the object. The stiffness and damping parameters of the virtual springs are set to be $220 \mathrm{~N} / \mathrm{m}$ and $100 \mathrm{Ns} / \mathrm{m}$, respectively. The results are illustrated in Fig. 3, which show the control forces increasing stably with decreasing distances between the fingers. 


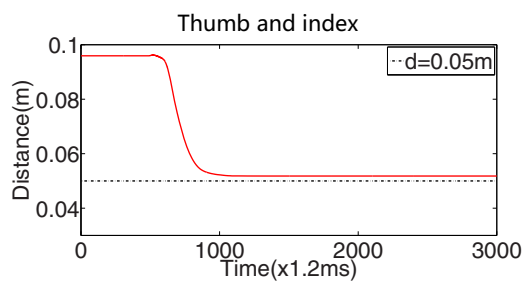

(a)

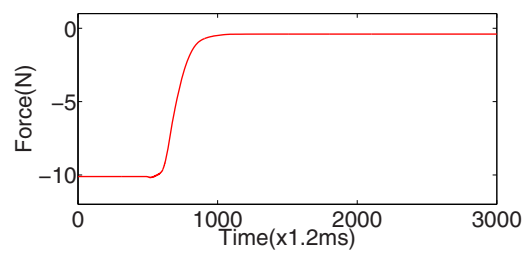

(c)

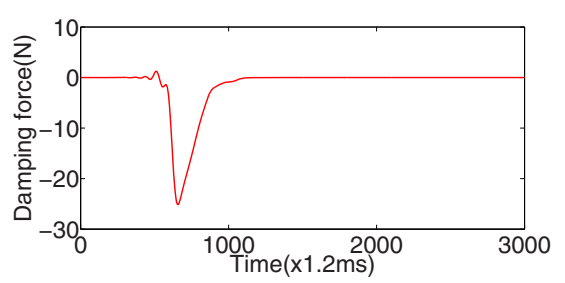

(e)

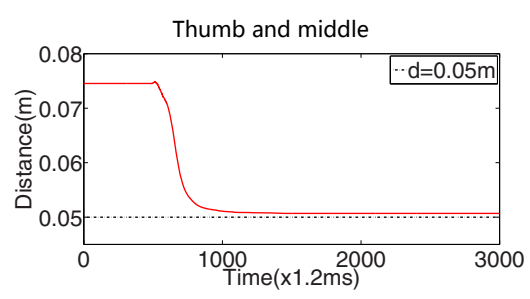

(b)

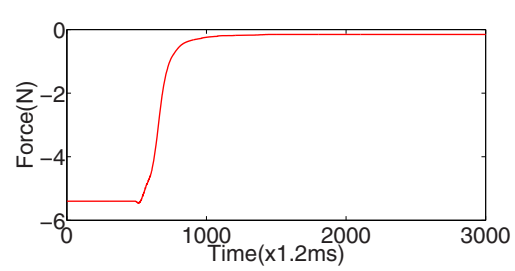

(d)

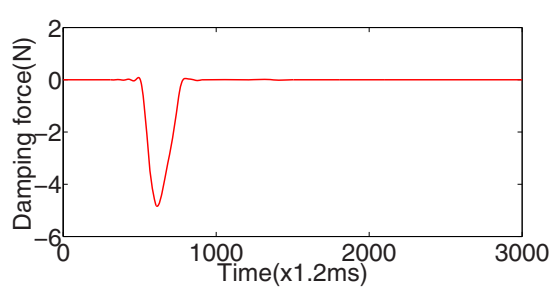

(f)

Fig. 3. (a) and (b) show the distance varying between thumb and index finger, thumb and middle finger, respectively. (c) and (d) demonstrate the spatial forces generated by the spatial springs of thumb-index and thumb-middle, with respect to the decreasing distance. (e) and (f) indicate the damping forces varying of the two pairs of fingers, respectively.

The rest length $(0.05 \mathrm{~m})$ of the thumb-index and thumb-middle finger pairs can be reached with the adaptive compliant grasp controller. The control error caused by the friction and gravity forces can be reduced with the friction and gravity compensation methods. The designed virtual spring frame as shown in Fig. 2 results in a significant higher force on the thumb than the remaining fingers, as it alone opposes four fingers.

The effectiveness of the self-collision avoidance virtual spring is shown as Fig. 4. The stiffness and damping parameters of the self-collision avoidance controller for the index-middle finger pair set at $160 \mathrm{~N} / \mathrm{m}$ and $80 \mathrm{Ns} / \mathrm{m}$, respectively. The rest length of the virtual spring is chosen to be $0.03 \mathrm{~m}$. The repelling force between the two fingers increases stably after the distance between the two fingers drop below the set threshold. In this way, the fingers are driven away from each other to avoid collision. 


\subsection{Grasp Experiments}

A large number of grasp trials have been carried out to assess the effectiveness of the adaptive compliant grasp approach control strategy. In all trials the robot fingers are commanded initially to achieve the expected object location, while the actual positions of the target object vary with different designated errors in both horizontal directions (X-and Y-axes). 10 grasp trails are carried out for each position error settings (e.g. position $(\mathrm{x}, \mathrm{y})=(-20 \mathrm{~mm}, 15 \mathrm{~mm}))$. Different finger combinations (2-, 3- and 5-finger form) with corresponding virtual spatial spring frames have been evaluated in the experiments. A cylindrical shaped glue stick $(\phi 30 \mathrm{~mm})$ has been used as the target object in the 2-finger form grasp task and 3 -finger form. A spray cleaner bottle $(\phi 50 \mathrm{~mm})$ has been used in the 5 -finger grasp task as the target object. A trial would be considered a success if the target object can be grasped and stably lifted up.

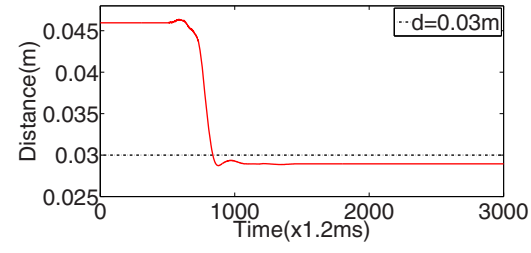

(a)

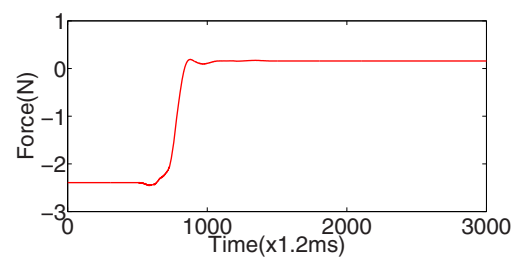

(b)

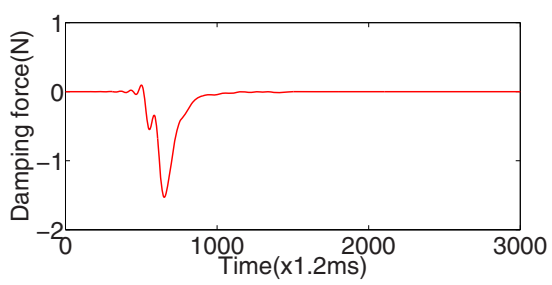

(c)

Fig. 4. (a) shows the distance varying between the two fingers. (b) and (c) demonstrate the repulsive force and damping response of the self-collision avoidance virtual spring with respect to changing distance.

The results of 2-, 3-, 5-finger grasp are shown in Fig. 5, which are illustrated as seen from point of view of above the target object. The green region is the area where successful object grasp and lift are achieved by both adaptive grasp approach and non-adaptive grasp control. The red region is the area where adaptive grasp approach succeeds in grasp and lift, while the non-adaptive grasp fails to accomplish the grasp task. The success rate is represented as the transparency rate of the area.

The experiments have shown that significantly larger position errors with respect to the hand workspace can be accommodated with the proposed adaptive 


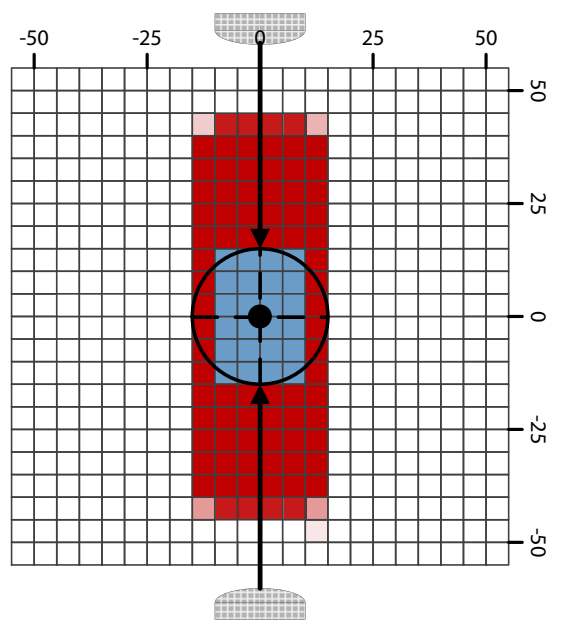

(a) two-finger grasp

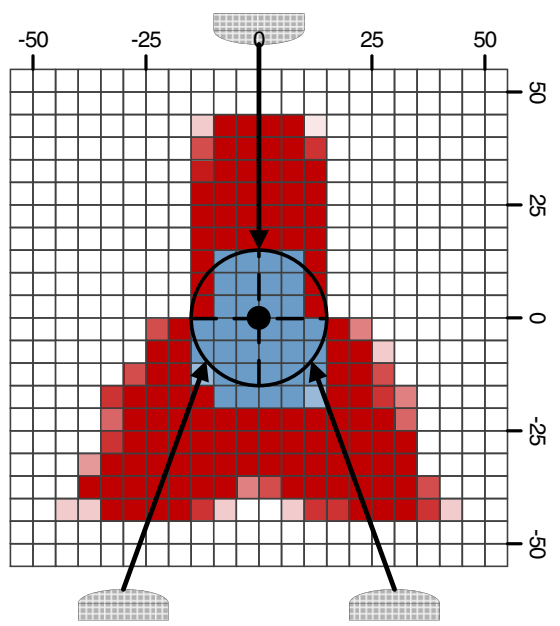

(b) three-finger grasp

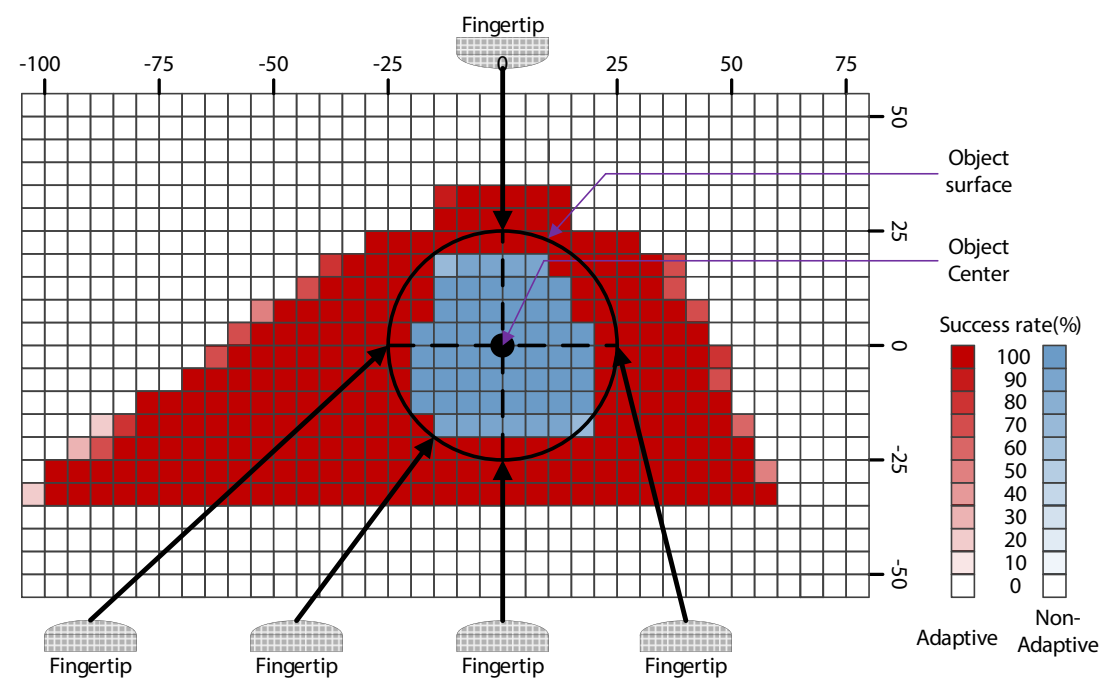

(c) five-finger grasp from the top

Fig. 5. The comparison of grasp success rates with adaptive compliant grasp versus non-adaptive grasp, all of which are for grasping the object from the top. The green region is the area where both grasp approach strategy succeed in grasping and lifting, while the red region is the area where only the adaptive grasp approach succeeds. The black circle represents the surface profile and expected position of the target object. The footprints of the fingertips are shown as gray rectangle-ellipse shape. The black arrows indicate the planned grasp approach trajectories of the robot fingertips. The two axes of the chart mark the actual position of the target with respect to the expected placement. 
compliant grasp control strategy. 246\%, 391\% and 333\% increase in position error area coverage have been obtained with 5-, 3-, and 2-finger form overhead grasp (approach from top), respectively. The results have been similarly successful for the 2-, 3-, and 5-finger side grasp trials (approach from side). It has been observed that a small position error of $15 \mathrm{~mm}$ can result in failure by non-adaptive grasps in 2-finger form and 3-finger form grasp. $20 \mathrm{~mm}$ position error can be accommodated by a 5 -finger grasp, thanks to the increasing finger numbers. On the other hand, the object can be displaced up to $45 \mathrm{~mm}$ in 2-finger and 3-finger grasp, and $35 \mathrm{~mm}$ in five grasp. Considering the open distance between the thumb and the remaining fingers $(120 \mathrm{~mm})$, and the size of the objects $(\phi 30 \mathrm{~mm}$ and $\phi 50 \mathrm{~mm})$, It has been deduced that the position uncertainties of the object have been accommodated along the planned trajectories of the fingertips, when using the adaptive grasp approach control strategy.

The non-symmetrical shape of the success regions during 3 -finger and 5 -finger grasps is a result of the non-symmetrical configuration of the fingers on the hand, where the thumb is designed to oppose to the remaining fingers, located in closest proximity to the index finger. The size of success region rises with increasing number of fingers utilized for the grasp. Experimental results indicate that the grasp performance improves with increasing number for fingers utilized for the grasp task. Conversely as the couplings and interferences between different fingers also increases with increasing number of fingers. Therefore, the adaptive compliant grasp control can also face a performance limit as a function of utilizing more fingers.

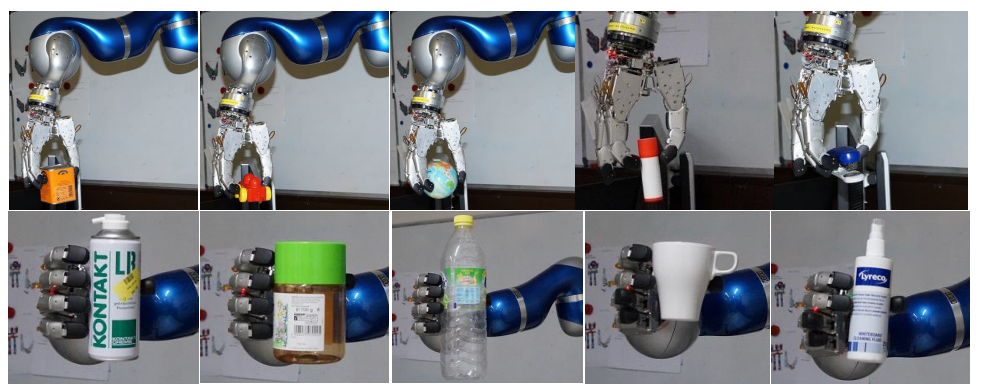

Fig. 6. Examples of successful grasps, from the side or above, of different objects with various object position errors.

The overall performance and robustness of the proposed strategy have been evaluated by grasping 10 representative dissimilarly shaped objects commonly found in ADL (activities of daily living), as shown in Fig. 6. Each object is placed on a table with a randomly selected position, constrained by the workspace of the robotic hand. 231 out of 240 attempts of grasping and lifting the object are stably achieved by adaptive compliant grasp approach, in comparison with 188 successful trials by non-adaptive grasp approach. Consistently higher success rates have been shown with the adaptive compliant grasp over a wide range of target objects and poses. 
Additionally, several observations have been made during the grasp experiments:

1. Thanks to the compliance behavior of proposed grasp controller, the grasped target objects can withstand some additional external disturbance, such as a light bump or a pull, and still have the grasp on the object maintained. The compliant, yet disturbance tolerant, grasp behavior once the object is in stable grip, not only provides more grasp robustness, but also benefits manipulation phases following Phase 5. This feature would be difficult to duplicate in a 2-jaw gripper or underactuated hand because of insufficient controllable degrees of freedom, sensor feedback and dynamic performance.

2. Although only local in-hand adjustment of the fingers have been considered in this paper, in cases where position errors of the target object have been beyond the workspace of robotic hand(where a re-grasp would be required), the proposed grasp strategy could be easily extended together with motion adjustment of robot arm.

3. The higher number of contact points at different position in space has given the multi-finger dexterous hand the ability to form a finger "basket" (particularly when using more fingers) to "catch" the object after it starts moving or tipping over due to contact with the object with a position error. This "catching" style of grasping the object, often used by humans, can be quite effective assuming sufficient available speed of robotic fingers.

\section{Conclusions and Future Work}

An adaptive compliant grasp control strategy based on a new interpretation of the spatial virtual spring for objects with position errors is proposed in this paper. The adaptive grasp control strategy is consisted of independent grasping phases, the grasp start phase, contact detection phase, reactive compliant control phase, adaptive compliant grasp phase, and grasp complete phase. During the adaptive grasp execution, the robot finger with the first unexpected impact remains in contact with the object, while the rest of the fingers are adaptively driven towards the adjusted grasping position without on-line replanning. Experimental results show robust and vastly improved grasp performance on objects with significantly higher tolerable positions errors of as much as $391 \%$, with the proposed adaptive compliant multi-fingered grasping control strategy. Finally, the full purpose of a dexterous hand is to manipulate the object after after grasping it. A strategy to address manipulation shall be extended into this grasp strategy pipeline going forward.

Acknowledgments. This work was partially funded by the EU project EuRoC (grant agreement no. CP-IP 608849).

\section{References}

1. Miller, A.T., Knoop, S., Christensen, H.I., Allen, P.K.: Automatic grasp planning using shape primitives. In: Proceedings of IEEE International Conference on Robotics and Automation, vol. 2, pp. 1824-1829 (2003) 
2. Lii, N.Y., Chen, Z., Roa, M.A., Maier, A., Pleintinger, B., Borst, C.: Toward a task space framework for gesture commanded telemanipulation. In: Proceedings of IEEE International Symposium on Robot and Human Interactive Communication, pp. 925-932. IEEE (2012)

3. Takahashi, T., Tsuboi, T., Kishida, T., Kawanami, Y., Shimizu, S., Iribe, M., Fukushima, T., Fujita, M.: Adaptive grasping by multi fingered hand with tactile sensor based on robust force and position control. In: Proceedings of IEEE International Conference on Robotics and Automation, Pasadena, CA, USA, pp. 264-271 (2008)

4. Hsiao, K., Chitta, S., Ciocarlie, M., Jones, E.G.: Contact-reactive grasping of objects with partial shape information. In: Proceedings of IEEE International Conference on Intelligent Robots and Systems, pp. 1228-1235 (2010)

5. Chalon, M., Reinecke, J., Pfanne, M.: Online in-hand object localization. In: Proceedings of IEEE International Conference on Intelligent Robots and Systems, pp. 2977-2984 (2013)

6. Hogan, N.: Impedance control-An approach to manipulation. I-Theory. II-Implementation. III-Applications. ASME Transactions on Dynamic Systems, Measurement, and Control 107 (1985)

7. Khatib, O.: Inertial properties in robotic manipulation: An object-level framework. The International Journal of Robotics Research 14(1), 19-36 (1995)

8. Fasse, E., Broenink, J.: A spatial impedance controller for robotic manipulation. IEEE Transactions on Robotics and Automation 13(4), 546-556 (1997)

9. Wimböck, T., Ott, C., Albu-Schäffer, A., Hirzinger, G.: Comparison of object-level grasp controllers for dynamic dexterous manipulation. The International Journal of Robotics Research 31(1), 3-23 (2012)

10. Stramigioli, S.: Modeling and IPC control of interactive mechanical systems: a coordinate-free approach, vol. 266. Springer, Germany (2001)

11. Birglen, L., Gosselin, C., Laliberté, T.: Underactuated robotic hands, vol. 40. Springer (2008)

12. Ciocarlie, M., Hicks, F.M., Stanford, S.: Kinetic and dimensional optimization for a tendon-driven gripper. In: Proceedings of IEEE International Conference on Robotics and Automation, pp. 2751-2758. IEEE (2013)

13. Tincani, V., Catalano, M.G., Farnioli, E., Garabini, M., Grioli, G., Fantoni, G., Bicchi, A.: Velvet fingers: a dexterous gripper with active surfaces. In: Proceedings of IEEE International Conference on Intelligent Robots and Systems, pp. 1257-1263 (2012)

14. Chen, Z., Lii, N.Y., Wimböck, T., Fan, S., Jin, M., Borst, C., Liu, H.: Experimental study on impedance control for the five-finger dexterous robot hand DLR-HIT II. In: Proceedings of the IEEE/RSJ International Conference on Intelligent Robots and Systems, IROS 2010, pp. 5867-5874. IEEE, Taipei (2010)

15. Liu, H., Wu, K., Meusel, P., Seitz, N., Hirzinger, G., Jin, M., Liu, Y., Fan, S., Lan, T., Chen, Z.: Multisensory five-finger dexterous hand: the DLR/HIT Hand II. In: Proceedings of IEEE/RSJ International Conference on Intelligent Robots and Systems, Nice, France, pp. 3692-3697 (2008)

16. Chen, Z., Lii, N.Y., Wimböck, T., Fan, S., Liu, H., Albu-Schäffer, A.: Experimental analysis on spatial and cartesian impedance control for the dexterous DLR/HIT II hand. International Journal of Robotics and Automation 29(1) (2014) 
17. Roa, M., Hertkorn, K., Borst, C., Hirzinger, G.: Reachable independent contact regions for precision grasps. In: Proceedings of IEEE International Conference on Robotics and Automation, pp. 5337-5343. IEEE, Shanghai (2011)

18. Albu-Schäffer, A., Ott, C., Hirzinger, G.: A passivity based Cartesian impedance controller for flexible joint robots-Part II: full state feedback, impedance design and experiments. In: Proceedings of IEEE International Conference on Robotics and Automation, New Orleans, LA, USA, pp. 2666-2672 (2004) 